\title{
Clinical usefulness of 18F-FDG PET in patients with hepatocellular carcinoma undergoing surgical resection
}

\author{
Min Ho SHIN, Nam Kyu CHOI*
}

Department of Hepatobililiary pancreas surgery, Chosun University Hospital, Gwangju, Korea

Introduction: The diagnosis and staging hepatocellular carcinoma (HCC) is important because of the different treatment methods and the prognosis. [18F]fludeoxyglucose positron emission tomography/computed tomography ([18F]FDG-PET/CT) has been suggested as a diagnostic modality in HCC. The aim of this study is to evaluate the accuracy of [18F]FDG-PET for staging of HCC after surgical resection and histological confirmation.

Methods: We retrospectively collected data of 56 patients who underwent [18F]FDG-PET before surgical resection for HCC from March 2011 to May 2017. All of whom were suitable for resection by conventional HCC staging. The results of maximal standardized uptake value (SUVmax) was compared with histological confirmation.

Results: A larger tumor size was related with a higher SUV $(\geq 4.9)$ and also serum alpha-feto protein was associated with SUV. The recurrence rate was higher in patients with a higher SUV and the patients with lower SUV had better survival rate.

Conclusions: HCC, [18F]fludeoxyglucose positron emission tomography, standardized uptake value (SUV) 